\title{
Co-registration of Histological, Optical and MR Data of the Human Brain
}

\author{
É. Bardinet ${ }^{1}$, S. Ourselin ${ }^{2}$, D. Dormont ${ }^{4}$, G. Malandain ${ }^{1}$, D. Tandé ${ }^{3}$, \\ K. Parain ${ }^{3}$, N. Ayache ${ }^{1}$, and J. Yelnik ${ }^{3}$ \\ 1 INRIA, Epidaure Project, Sophia Antipolis, France \\ 2 CSIRO Telecom. and Industrial Physics, PO Box 76, Epping NSW 1710 Australia \\ 3 INSERM U289, Salpêtrière Hospital, France \\ 4 Neuroradiology Dept and LENA UPR 640-CNRS, Salpêtrière Hospital, France \\ ebard@sophia.inria.fr
}

\begin{abstract}
In order to allow accurate pre-operative localisation of functional targets in functional neurosurgery, we aim at constructing a three dimensional registrable cartography of the basal ganglia, based on histology. For doing this, a post mortem MR study was conducted on a cadaver's head, and the brain was then extracted and processed for histology. The post mortem MR image will allow to report the cartography on the patient's anatomy, by its registration with the patient's MR image. In this paper, we focus on the problem of co-registering the histological and post mortem MR data of the same subject. First, realignment of the histological sections into a reliable three dimensional volume is performed. Then the reconstructed volume is registered with the post mortem MR image. To insure three dimensional integrity of the histological reconstructed volume, a reference volume is first constructed from photographs of the unstained surface of the frozen brain. This reference is then used as an intermediate volume for, on the one hand, independant alignment of each histological section with its corresponding optical section and on the other hand, three dimensional registration with the post mortem MR image.
\end{abstract}

\section{Introduction}

Advances in image guided stereotactic neurosurgery and stimulation technology have given rise to a reappearence of the use of functional neurosurgery for the treatment of movement disorders, e.g. Parkinson's disease or dystonia. The intervention is based on the stereotactic introduction of electrodes in disease-specific nuclei of the basal ganglia, e.g. for Parkinson's disease a small, deeply located, nucleus called the subthalamic nucleus (STN) 6]. The nucleus is targeted on pre-operative stereotactic MR acquisitions [1]. In these procedures, the surgical success depends primarily on the accurate localisation of the target.

MR imaging of the basal ganglia, despite technological and clinical progress over the last decade, appears to be intrinsically limited by two factors. First, the resolution of clinical routine $\mathrm{MR}$ images is nowadays around $1 \mathrm{~mm}^{3}$, thus limiting the level of detail of the images to the gross features of the basal ganglia;

T. Dohi and R. Kikinis (Eds.): MICCAI 2002, LNCS 2488, pp. 548 5552002.

(C) Springer-Verlag Berlin Heidelberg 2002 
second, and more fundamentally, the MR signal, being a measure of tissue physical properties, provides us with a representation of the underlying anatomical reality of the organ being imaged. The relationship between the measured signal and the real anatomy is not always completely understood. For example, for Parkinson's disease, the STN is targeted on pre-operative MR acquisitions (T1 and T2-weighted) [1. On the T1 image (acquired in stereotactic conditions), this nucleus is undistinguishable, and on the T2 image (not in stereotactic conditions, due to geometric distortions), an hyposignal at the STN level is observed, but a clearly defined relationship between this signal and the STN is still debated. This introduces uncertainty on the nucleus localisation. Consequently, electrophysiological study and clinical testing are performed during the intervention to refine the pre-operatively determined target position. This causes the intervention to last on average 10 hours. Therefore, allowing more accurate pre-operative locatisation of the functional targets appears to be a key issue.

Detailed and accurate cartography of the basal ganglia can be performed on histological serial sections. Indeed, histology, consisting in the study of postmortem autopsy tissues, overcomes the limitations of MR imaging, allowing higher level of detail and direct observation of anatomical reality [117]. Moreover, staining of histological sections can provide functional information, e.g. Nissl stain which can reveal cytoarchitectonic details of cerebral regions, or Calbindin immunoreactivity which can distinguish the associative and sensori-motor territories of the striatum [2]. Therefore, anatomical and functional cartography of the complete basal ganglia can be performed on histology, and the corresponding features accurately outlined. Nevertheless, before to use such a cartography for target localisation in functional neurosurgery, reliable three dimensional reconstruction from the histological sections must be done, as histology being by nature two-dimensional, i.e. histological data consist of a series of discontinuous serial sections. Also, this cartography has to be registrable with the MR acquisitions of the patient, in order to report the outlined features on the patient's anatomy.

Resuming, we aim at constructing a three dimensional, anatomical and functional, as well as registrable, cartography of the basal ganglia, based on histology. For doing this, a post mortem MR study was conducted on a cadaver's head, 36 hours after death, insuring the MR signal to be very similar to an in vivo MR image, and the brain was then extracted and processed for histology. The post mortem MR image will allow to report the cartography on the patient's anatomy, by its registration with the patient's MR image.

In this paper, we focus on the problem of co-registering histological and post mortem MR data of the same subject. First, realignment of the histological sections into a reliable three dimensional volume has to be performed. Then this reconstructed volume has to be registered with the MR image. Because histological sections suffered independant two dimensional geometric distortions, direct alignment of these sections would not allow to get a reliable three dimensional volume, lacking a three dimensional reference. In order to build such a reference, photographs were taken during brain sectionning. Their alignment provides us with a reference volume, which is then used as an intermediate volume for, on the one hand, alignment of the histological sections with their corresponding optical sections, and on the other hand, three dimensional registration with the post mortem MR image. 

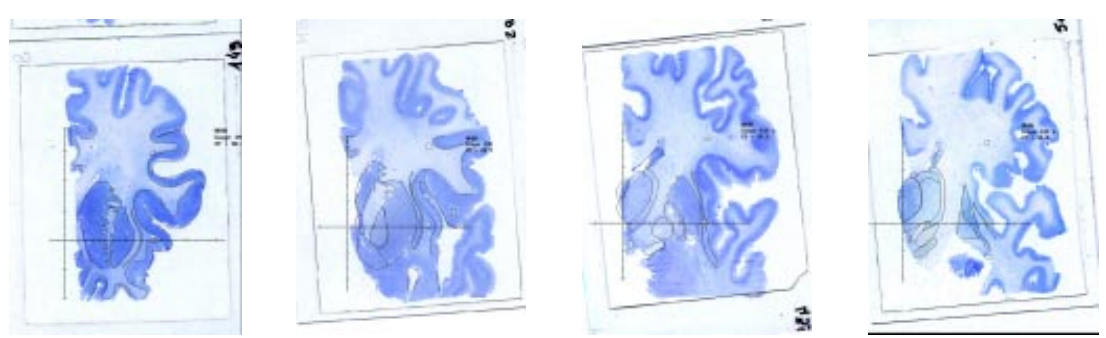

Fig. 1. Nissl stained histological sections with outlines of basal ganglia structures and territories superimposed.

\section{Material and Methods}

\subsection{Material}

A human brain was obtained 36 hours after death. First an MR study (T1 and T2-weighted acquisitions) was conducted, before extraction of the brain. Then the brain was stored in $4 \%$ paraformaldehyde for 8 days and in phosphate buffer with sucrose for 7 days. One hemisphere was sectioned into 3 blocks $(1.5 \mathrm{~cm}$ thick) in order to favour a better fixation, and stored frozen at $-40^{\circ} \mathrm{C}$. The blocks were cut into $70 \mu \mathrm{m}$ thin sections which were collected serially. Sectioning was done on a Tetrander Jung freezing microtome. During cutting, some sections at the bottom and top of the blocks were lost as the cryomicrotome could not reach them. Sections were treated according to different immunohistochemical procedures. One out of ten sections (thus every $700 \mu \mathrm{m}$ ) was stained for Nissl to reveal cytoarchitectonic details of cerebral regions. After staining, histological sections were scanned, and structures and territories of the basal ganglia were outlined (Figure 1). During sectioning, photographs of the unstained surface of the frozen brain, together with part of the cryomicrotome including 6 screws, were taken for one out of ten sections (Figure 2). There was therefore a corresponding optical section for each histological stained section.

\subsection{Fusion of Histological and Post Mortem MR Data}

As explained in section 11. realignment of the histological sections has to be performed first, followed by the registration of the reconstructed volume with the post mortem MR image.

Alignment of histological serial sections into a three dimensional volume can be done following a straightforward method. It consists in the registration of consecutive sections two by two and further three dimensional reconstruction by composition of the resulting transformations [4359]. Nevertheless, this method is not adequate when the sections have suffered independant two dimensional geometric distortions. Indeed, rigid registration of contiguous sections would not compensate for the distortions, and non rigid registration, which could partially cope with distortions, followed by transformation composition, would not allow to get a reliable three dimensional histological volume in the absence of a three dimensional reference.

In order to build such a reference, following [128, photographs of the unstained surface of the frozen brain were taken. Feature-based alignment of these 

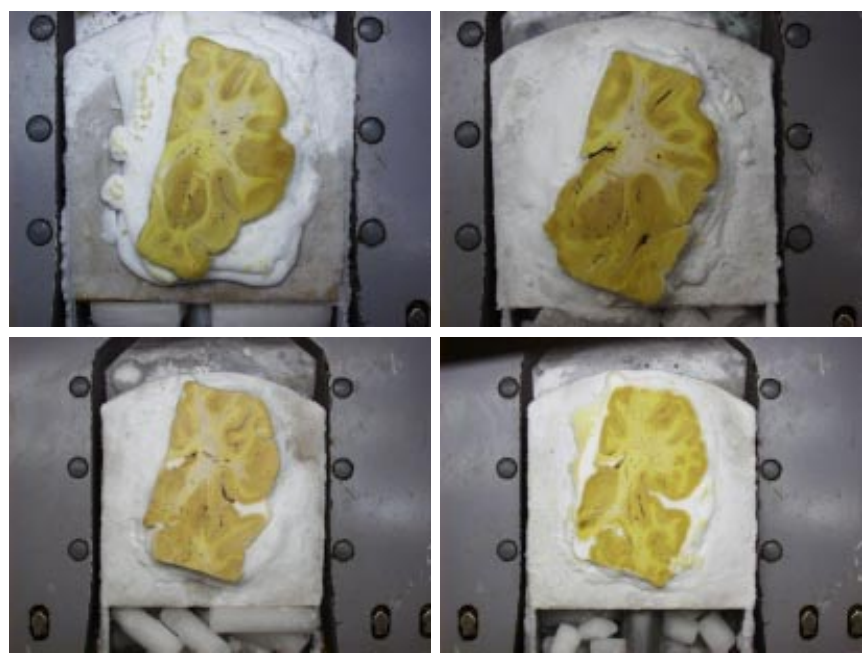

Fig. 2. Photographs of the unstained surface of the frozen brain taken during brain sectioning, together with part of the cryomicrotome including 6 screws.

photographs, the screws of the cryomicrotome acting as fiducial markers, yields a reference volume. This reference is then used as an intermediate volume for, on the one hand, independant alignment of each histological section with its corresponding optical section (this guarantees the three dimensional integrity of the reconstructed histological volume) and on the other hand, three dimensional registration with the post mortem MR image.

Feature-based alignment of the optical sections. In order to reconstruct a reference three dimensional volume from the optical sections, we used a featurebased registration method (rigid ICP - Iterative Closest Point algorithm), where the 6 screws of the cryomicrotome served as fiducial markers. These screws were detected automatically by combination of thresholding and connected component analysis. Alignment of the optical sections using these markers guaranteed the integrity of the resulting three dimensional reconstruction. The resulting volume presented intensity variations from slice to slice, due to the photographs acquisition and scanning process. In order to get an homogeneous volume, these variations were corrected by histogram equalisation.

Reconstruction of the histological volume. Using the optical reconstructed volume as a geometrical reference, each histological section was registered with its corresponding optical section. The $2 \mathrm{D}$ registrations were performed with the block matching algorithm [10], an intensity-based two-steps method consisting in selective local-based correspondance computation, followed by robust transformation estimation, these two steps being embedded in an iterative multi-scale scheme (typically 6 levels). Correlation coefficient was used as the similarity measure for computation of the correspondances, and 2D affine transformations, one for each section, were estimated.

Selective local-based correspondance computation and robust estimation of the transformation made this algorithm particularly adapted to the problem, capable to cope with distortions due to the processing of the histological sections. Indeed, most of the correspondances were found in highly contrasted regions, 

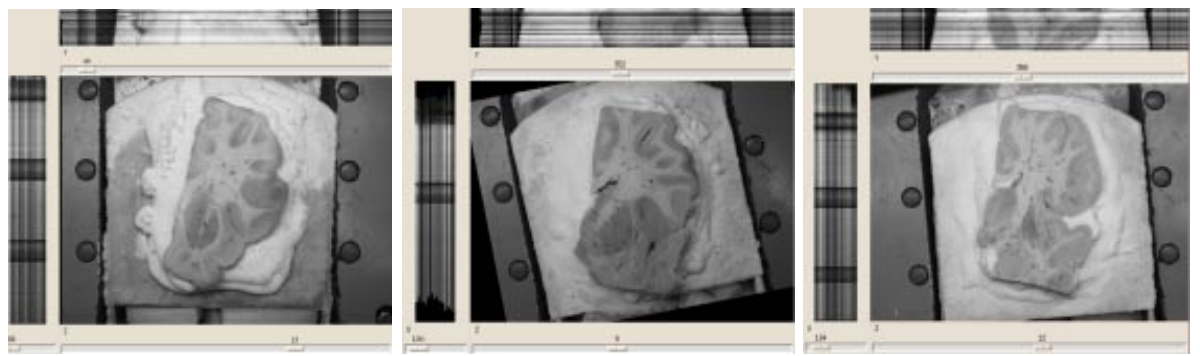

Fig. 3. Rigid alignment of the optical sections. Three orthogonal views of each of the three brain blocks (see text for details)

i.e. around the basal ganglia, and irrelevant correspondances were considered as outliers during robust estimation.

Registration with the post mortem MR data. To achieve the fusion of histology and MR data, the remaining step consisted in registering in 3D the intermediate reference optical volume with the T1-weighted post mortem MR image. Again, this registration was performed with the block matching algorithm [10], and a unique three dimensional affine transformation was estimated.

\section{Results and Discussion}

First, feature-based alignment of the optical sections was performed. As explained in section [2.1] the brain (one hemisphere) was first sectioned into 3 blocks $(1.5 \mathrm{~cm}$ thick) in order to favour a better fixation, before histological sectioning in thin sections. Consequently, photographs taken during sectioning consisted in three series, for which feature-based registration, using cryomicrotome screws as fiducial markers, lead to reliable three dimensional alignments (three orthogonal views of the alignments are shown Figure 3- note the alignment of the cryomicrotome on lateral views, as well as intensity variations from slice to slice). To get the complete optical volume, alignment of the last section of the first (resp. second) block with the first section of the second (resp. third) block was then performed manually by an expert, to insure perfect structural continuity between the blocks around the basal ganglia. Alignment of the complete optical sections followed by correction of intensity variations can be seen in Figure 5 (left).

Then, each histological section was independantly registered with its corresponding optical sections. On Figure 4, some results are presented: optical sections (first row), registered corresponding histological sections (second row), and both sections fused (third row), in order to visually assert the quality of the registrations. Stacking-up the registered histological sections lead to a reliable three dimensional histological volume (Figure [5] right). As said in section 2.1] some histological sections at the top and bottom of the blocks were lost during brain cutting. The two gaps in the sagittal and axial views of the reconstructed histological volume on Figure 5 correspond to these missing sections.

Registration of the reference optical volume with the T1-weighted post mortem MR image was finally performed, as well as registration of the T1 and T2weighted MR images. 

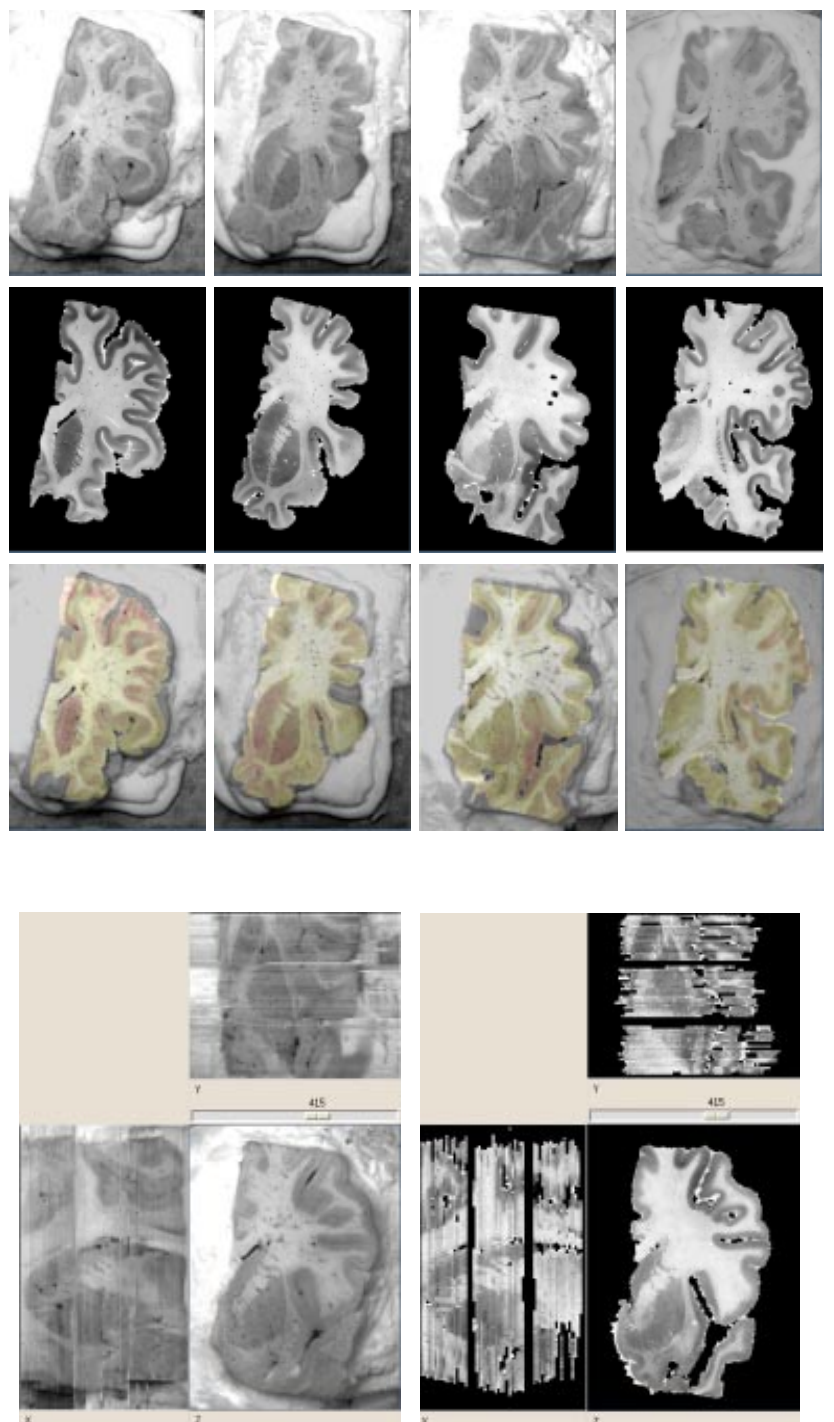

$x$
Fig. 4. Two dimensional registration of histological and optical sections. First row: four optical sections along the anteroposterior axis; second row: corresponding histological sections after registration; third row: both sections fused with an opacity factor, allowing to visually assert the good correspondence for the basal ganglia.

Fig. 5. Three orthogonal slices through the optical (left) and histological (right) reconstructed volumes (see text for details).

For registration of the histological sections as well as fusion of the optical volume with the T1-weighted MR image, our interest being the basal ganglia, the block matching algorithm was particularly adapted, as correspondances were found in highly contrasted regions, i.e. around the basal ganglia, and irrelevant correspondances were considered as outliers during robust estimation of the transformations.

Once co-registration of the histological, optical and MR data was achieved, we reported structures and territories of the basal ganglia outlined on the histological Nissl-stained sections onto the histological, optical and post mortem MR 

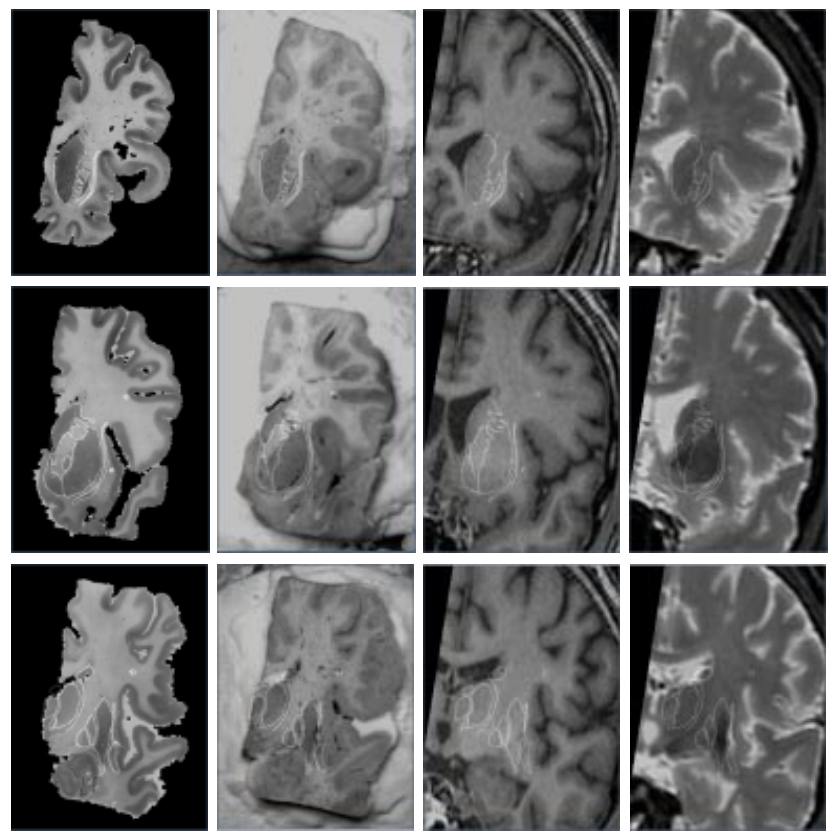

Fig. 6. Histological, optical and post mortem ( $\mathrm{T} 1$ and T2-weighted) MR volumes co-registered. Four sections along the anteroposterior axis. Structures of the basal ganlia outlined on the histological sections are reported
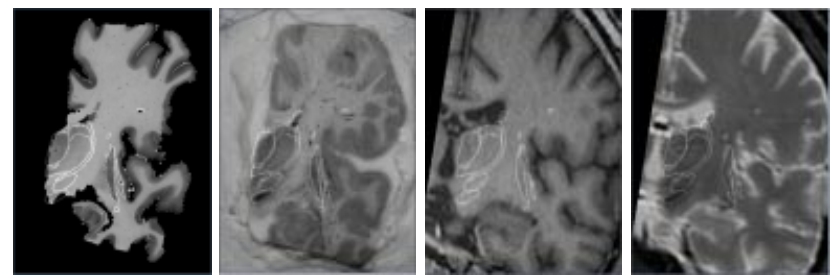

on the four volumes (including caudate nucleus, putamen, pallidum, claustrum, accumbens, nuclei of the thalamus, subthalamic nucleus and substancia nigra).

volumes using the corresponding transformations. On Figure 6, four sections of these volumes, along the antero-posterior axis, are shown, with contours of the basal ganlia superimposed (including caudate nucleus, putamen, pallidum, claustrum, accumbens, nuclei of the thalamus, subthalamic nucleus and substancia nigra).

\section{Conclusion}

In this study, we have carried out co-registration of histological and post mortem MR data of the same subject. This work is part of a project which aims at constructing a three dimensional, anatomical and functional, as well as registrable, cartography of the human basal ganglia, based on histology, in order to improve accuracy of the pre-operative localisation of targets in functional neurosurgery.

Realignment of the Nissl-stained histological sections into a reliable three dimensional volume was first performed. Then the reconstructed volume was registered with the post mortem MR image. To insure three dimensional integrity of the histological reconstructed volume, an intermediate reference volume was first constructed from photographs of the unstained surface of the frozen brain. 
Co-registration of the histological, optical and MR data allowed us to report structures and territories of the basal ganglia outlined on the histological sections onto the post mortem MR volumes.

In order to use this three-dimensional cartography of the basal ganglia for pre-operative planning in functional neurosurgery, the remaining step consists in an accurate registration of the post mortem MR data with the patient's MR data, that will allow to report this cartography on patient's anatomy.

\section{References}

1. B.P. Bejjani, D. Dormont, B. Pidoux, J. Yelnik, P. Damier, and I. Arnulf. Bilateral subthalamic stimulation for parkinson's disease by using three-dimensional stereotactic magnetic resonance imaging and electrophysiological guidance. Journal of Neurosurgery, 92:615-625, 2000.

2. C. François, J. Yelnik, G. Percheron, and D. Tandé. Calbindin d-28k as a marker for the associative cortical territory of the striatum in macaque. Brain Research, 633:331-336, 1994.

3. E. Guest and R. Baldock. Automatic reconstruction of serial sections using the finite element method. Bioimaging, 3:154-167, 1995.

4. L.S. Hibbard and R.A. Hawkings. Objective image alignment for three-dimensional reconstruction of digital autoradiograms. Journal of Neuroscience Method, 26:5574, 1988.

5. B. Kim, J.L. Boes, K.A. Frey, and C.R. Meyer. Mutual Information for Automated Unwarping of Rat Brain Autoradiographs. Neuroimage, 5:31-40, 1997.

6. P. Limousin, P. Pollak, D. Benazzouz, D. Hoffmann, J.F. Le Bas, E. Broussolle, J.E. Perret, and A.L Benabid. Effect of parkinsonian signs and symptoms of bilateral subthalamic nucleus stimulatiom. Lancet, 345, 1995.

7. J. Mai, J. Asscheuer, and G. Paxinos. Atlas of the Human Brain. Academic Press, 1997.

8. M.S. Mega, S.S. Chen, P.M. Thompson, R.P. Woods, T.J. Karaca, A. Tiwari, H.V. Vinters, G.W. Small, and A.W. Toga. Mapping Histology to Metabolism: Coregistration of Stained Whole-Brain Sections to Premortem PET in Alzheimer's Disease. Neuroimage, 5:147-153, 1997.

9. S. Ourselin, E. Bardinet, D. Dormont, G. Malandain, A. Roche, N. Ayache, D. Tande, K. Parain, and J. Yelnik. Fusion of histological sections and MR images: towards the construction of an atlas of the human basal ganglia. In W.J. Niessen and M.A. Viergever, editors, 4th Int. Conf. on Medical Image Computing and Computer-Assisted Intervention (MICCAI'01), volume 2208 of LNCS, pages 743751, Utrecht, The Netherlands, October 2001.

10. S. Ourselin, A. Roche, S. Prima, and N. Ayache. Block Matching: A General Gramework to Improve Robustness of Rigid Registration of Medical Images. In A.M. DiGioia and S. Delp, editors, International Conference on Medical Image Computing And Computer-Assisted Intervention, pages 557-566, Pittsburgh, Pennsylvania USA, October 11-14 2000.

11. G. Schaltenbrand and W. Wharen. Atlas for Stereotaxy of the Human Brain. Stuttgart: Georg Thieme Verlag, 1977.

12. T. Schormann, M. Von Matthey, A. Dabringhaus, and K. Zilles. Alignment of 3D Brain Data Sets Originating From MR and Histology. Bioimaging, 1:119-128, 1993. 